\title{
Valor pronóstico de los marcadores bioquímicos en pacientes con COVID-19
}

\author{
Prognostic value of biochemical markers in patients with COVID-19 \\ Jennifer Carolina Gutiérrez Suárez ${ }^{1}$, Carmen Cecilia Almonacid Urrego², \\ Hernández Rojas Edith del Carmen ${ }^{3}$, Hugo Mendieta Zerón. Ph. D. ${ }^{4}$
}

\section{Resumen}

El SARS-CoV-2 es un virus de la familia Coronaviridae, subfamilia coronavirus (CoV) y género $\beta$. Este se ha convertido en una amenaza inminente para toda la humanidad por ser el agente causal de la pandemia COVID-19, la cual llevó, por un lado, a la declaratoria de emergencia sanitaria a nivel mundial por parte de la Organización Mundial de la Salud (OMS) y, por otro, a instituir estrictas medidas de control para prevenir su contagio por parte de muchos gobiernos. En cuanto a la fisiopatología presentada en esta entidad, aunque las lesiones pulmonares han sido consideradas como las principales consecuencias de esta infección, a medida que avanza el conocimiento sobre el virus se han identificado también lesiones a nivel cardiaco, hepático y renal, que potencian la severidad de la infección y generan un mayor deterioro de los pacientes, su ingreso a las Unidades de Cuidados Intensivos y un mayor riesgo de mortalidad. Con base en esto, diversas investigaciones se han encaminado a determinar aquellos hallazgos clínicos y paraclínicos que puedan ser relevantes frente al pronóstico de los pacientes. Por lo anterior, la presente revisión aborda literatura disponible sobre los principales biomarcadores bioquímicos reportados por su asociación a daños cardiaco, hepático y renal, los cuales presentan mayor significancia para

\footnotetext{
1. M. Sc. en Ciencias Farmacéuticas, bacterióloga y laboratorista clínica. Grupo ECZA, Facultad de Ciencias de la Salud, Universidad Colegio Mayor de Cundinamarca, Colombia. ORCID: https://orcid.org/0000-0002-6597-6368

2. Ph. D. Biomedicina M. Sc. Microbiología, con énfasis en Bioquímica, bacterióloga y laboratorista clínica. Grupo ECZA, Facultad de Ciencias de la Salud, Universidad Colegio Mayor de Cundinamarca, Colombia. Asociación Científica Latina (ASCILA).

ORCID: https://orcid.org/0000-0002-0218-9367

3. Bacterióloga, M. Sc. Ciencias Microbiología con énfasis en Biología Molecular. Grupo de Investigación ECZA, Facultad de Ciencias de la Salud, Universidad Colegio Mayor de Cundinamarca. Grupo INSAFUSM, Facultad de Ciencias de la Salud, Fundación Universitaria San Martin. Asociación Científica Latina (ASCILA).

ORCID: https://orcid.org/0000-0002-2874-068X

4. Facultad de Medicina, Universidad Autónoma del Estado de México (UAEMéx), Grupo de Investigación ECZA. Asociación Científica Latina (ASCILA).

ORCID: https://orcid.org/0000-0003-3492-8950
}

Correo electrónico de correspondencia: jcarolinagutierrez@unicolmayor.edu.co 
evaluar el curso, severidad, manejo y pronóstico de la infección, y cuya alteración conlleva finalmente a un mayor riesgo de mortalidad en pacientes hospitalizados que presentan COVID-19.

Palabras claves: diagnóstico, COVID-19, biomarcadores, rastreo, biotransformación, metabolismo.

\section{Abstract}

SARS-CoV-2 is a virus from the coronaviridae family, coronavirus (CoV) subfamily and genus $\beta$, it has become an imminent threat to all humanity as it is the causal agent of the COVID-19 pandemic, which led to On the one hand, the World Health Organization (WHO) declares a worldwide health emergency, and on the other, to institute strict control measures to prevent its spread by many governments. Regarding the pathophysiology presented in this entity, although lung lesions have been considered the main consequences of this infection, as knowledge about the virus progresses, cardiac, hepatic, and renal lesions have also been identified that enhance severity of the infection generating greater deterioration of the patients, their admission to the Intensive Care Units and a higher risk of mortality; Based on this, various investigations have aimed to determine those clinical and paraclinical findings that may be relevant to the prognosis of the patients. Therefore, this review addresses available literature on the main biochemical biomarkers reported for their association with cardiac, liver and kidney damage, which are more significant in evaluating the course, severity, management and prognosis of the infection and whose alteration ultimately leads to an increased risk of mortality in hospitalized patients presenting with COVID-19.

Keywords: Diagnostic, COVID-19, Biomarkers, Screening, Biotransformation, Metabolism. 


\section{Marcadores cardiovasculares}

Los hallazgos asociados a mortalidad en pacientes con COVID-19 incluyen miocarditis fulminante explicada por dos mecanismos: tormenta de citoquinas manifestada por niveles elevados de interleucina-6 (IL-6), ferritina, lactato deshidrogenasa (LDH) y dímero D o un efecto directo del síndrome respiratorio agudo severo del virus sobre el corazón; sin embargo, el mecanismo directo de la acción del virus aún se encuentra en investigación $(1,2)$. De igual manera, se ha evidenciado la asociación de la infección con enfermedad cardiovascular (ECV) (1), que se describen a continuación.

Con respecto a las troponinas cTnI y cTnT, dos estudios (incluyendo un metaanálisis que incluyó 4 estudios reuniendo una población de 341 pacientes) evidenciaron niveles elevados en pacientes con COVID-19, en algunos casos con incrementos por encima de percentil 99 (>28 $\mathrm{pg} / \mathrm{ml}$ ), que se asociaron a una mayor severidad en los síntomas y presentación de lesión miocárdica $(3,4,5)$. Asimismo, niveles altos de cTnI se han correlacionado con cifras de mortalidad superiores al 50 $\%(6-8)$.

Si bien existe una mayor tasa de mortalidad cuando el paciente, además de presentar ECV tiene niveles aumentados de cTnT (9), hay un alto porcentaje de pacientes que exhiben incrementos en cTnT sin padecer ECV concomitante, $(1,7,10)$.
Sin embargo, y a pesar de estas evidencias, algunos autores afirman que no es posible establecer asociación entre los niveles de troponina y el índice de mortalidad $(11,12)$.

Para el caso de la proteína $\mathrm{C}$ reactiva (PCR), niveles por encima de los 10 $\mathrm{mg} / \mathrm{L}$ se han asociado con mayor severidad en la presentación de la infección por el SARS-CoV-2 (8,9,13-21), e incluso se han correlacionado con lesión pulmonar severa (22). Asimismo, se ha evidenciado una relación directa entre los valores de troponina y la PCR con la gravedad de la inflamación $(2,7,10,19,20)$ y la lesión miocárdica $(13,23)$.

En relación con el péptido natriurético $B$ $(\mathrm{PNB})$ y la creatina quinasa isoforma cardiaca (CKMB), estos tienen un inferior valor pronóstico que las troponinas en la patología cardiaca asociada a pacientes con SARS-CoV-2 (3,7,12,23,24-29).

En cuanto a los marcadores de coagulación, todos los estudios publicados demuestran un incremento en los valores de dímero $\mathrm{D}$ $(>0,5 \mathrm{mg} / \mathrm{l})(1,3,6,9,12-14,19,25,27-35)$, mientras que solo algunos revelan prolongación en el tiempo de protrombina (PT $>12$ s) $(1,3,8,14,19,25,29)$ y niveles decrecientes de fibrinógeno (23). En todos los casos, los niveles anormales de estos marcadores, en el ingreso de los pacientes, se asociaron con lesión cardíaca aguda, lo que implica que se correlacionan con la lesión miocárdica y la gravedad de 
la infección. Asimismo, se postula que la continua activación de la coagulación a lo largo del curso clínico de los no sobrevivientes puede ayudar a identificar pacientes deteriorados que requieren más apoyo o cuidados paliativos $(18,19,20,24,25)$.

Con respecto a las plaquetas, unos estudios no observaron diferencias significativas $(3,24,27,31,35)$, y otros reportaron trombocitopenia en pacientes con mayor severidad de la infección e ingreso a la UCI $(3-6,9,12,14,24)$, aunque los datos no son conducentes como sí lo son para el dímero $\mathrm{D}(10,13,25)$.

Para el caso particular de la angiotensina II, se identificó una relación directa entre los niveles aumentados de esta molécula frente a la carga viral y el daño en el tejido pulmonar de los pacientes con COVID-19 $(23,24,36)$.

\section{Marcadores hepáticos}

Aunque en algunos estudios no encontraron cambios significativos en la alanino aminotransferasa (ALT) y aspartato aminotransferasa (AST) $(10,16,20,37)$, la mayoría de los hallazgos demuestran incrementos superiores a $80 \mathrm{U} / \mathrm{L}$ que se relacionan de forma directa con la severidad de la infección, el UCI y la tasa de mortalidad de los pacientes, lo que además revela un deterioro hepático inminente $(13,16,27,31,37-39)$.
En pacientes con presentación severa de la enfermedad, también se han encontrado incrementos en los niveles de los marcadores colestásicos bilirrubinas totales, gama glutamil transpeptidasa y fosfatasa alcalina $(14,27)$. Todo ello corrobora que el SARS-CoV-2 no solo infecta y daña las células hepáticas, sino que causa disfunción del conducto biliar, con lo cual se postula que la tormenta de citoquinas causada por una respuesta inmune excesiva inducida por el virus puede ser una de las vías del daño hepático (39).

\section{Marcadores de daño renal}

La nefropatía es una complicación importante de COVID-19 y un factor de riesgo significativo de muerte (10). Aunque las concentraciones de creatinina y alfa-hidroxibutirato deshidrogenasa son más altas en pacientes con infección severa $(>90 \mu \mathrm{mol} / \mathrm{L})$, la diferencia no es estadísticamente significativa al compararlas con pacientes con sintomatología leve $(10,11,27,29)$. Sin embargo, la relación establecida entre los incrementos en creatinina $(>99 \mu \mathrm{mol} / \mathrm{L})$ y las concentraciones de proteinuria y hematuria en pacientes hospitalizados pone de manifiesto una insuficiencia renal con probabilidad más alta de ingreso a UCI y mayor riesgo de deterioro de los pacientes (10). De igual manera, la fuerte relación encontrada entre la severidad de la enfermedad y los aumentos en los niveles de alfa-hidroxibuti- 
rato deshidrogenasa $(>540 \mathrm{U} / \mathrm{L})$ predice el ingreso a UCI y la tasa de mortalidad de los pacientes (13).

\section{Otros marcadores}

Se han demostrado alteraciones en lactato deshidrogenasa $(\mathrm{LDH})$, proteínas totales y procalcitonina (PCT). Incrementos en $\mathrm{LDH}$ (incluso por encima de $600 \mathrm{U} / \mathrm{L}$ ), se han asociado con mayor severidad en la infección, mayor probabilidad de ingresar a UCI y mayor riesgo de fallecer $(1,13,14,16,18,20-22,27,37,40-44)$. Asimismo, se han relacionado de manera directa con la insuficiencia renal (13).

En este tipo de pacientes se observa, también, disminución en los niveles de proteínas totales (menor a $60 \mathrm{~g} / \mathrm{L}$ ), especialmente de la albúmina $(13,14,18,27)$, lo que obliga a mantener un mayor índice nutricional durante la hospitalización, que contribuya a incrementar las proteínas y disminuir la severidad de la infección (13).

Aunque la mayoría de los pacientes presentan niveles normales de este PCT (45-47), se ha observado que se pueden incrementar en aquellos que presentan coinfección $(8,32)$.

Aunque, debido al corto tiempo de inicio de la pandemia, la investigación en este campo es incipiente, todo lo anterior pone de manifiesto la necesidad de establecer perfiles de riesgo y diagnóstico que involucren la medición de biomarcadores para el manejo y pronóstico de pacientes con COVID-19 (48).

\section{Referencias}

1. Clerkin K, Fried J, Raikhelkar J, Sayer G, Griffin J, Masoumi A, et al. COVID-19 and Cardiovascular Disease. Circulation. 2020 [citado 17 de junio de 2020]; 141:1648-1655 DOI: https://doi. org/10.1161/CIRCULATIONAHA.120.046941

2. Chen C, Zhou Y, Wang DW. SARS-CoV-2: a potential novel etiology of fulminant myocarditis. Herz. 2020;45: 230-232. DOI: https://doi.org/10.1007/ s00059-020-04909

3. Huang Ch, Wang Y, Li X, Ren L, Zhao J, Hu Y, et al. Clinical features of patients infected with 2019 novel coronavirus in Wuhan, China. The Lancet. 2020;395:497-506. DOI: https://doi.org/10.1016/ S0140-6736(20)30183-5

4. Lippi G, Lavie CJ, Sanchis-Gomar F. Cardiac troponin I in patients with coronavirus disease 2019 (COVID-19): Evidence from a meta-analysis. Prog Cardiovasc Dis. 2020 May-Jun;63(3):390-391. DOI: 10.1016/j.pcad.2020.03.001. Vestjens SMT, Spoorenberg SMC, Rijkers GT, et al. High-sensitivity cardiac troponin $\mathrm{T}$ predicts mortality after hospitalization for community-acquired pneumonia. Respirology. 2017;22(5):1000-1006. DOI: https:// doi.org/10.1111/resp.12996

5. Shi S, Qin M, Shen B, Cai Y, Liu T, Yang F, Gong W, Liu X, Liang J, Zhao Q, Huang H, Yang B, Huang C. Association of Cardiac Injury With Mortality in Hospitalized Patients With COVID-19 in Wuhan, China. JAMA Cardiol. 2020;5(7):802-10. DOI: https://doi.org/10.1001/jamacardio.2020.0950 
G Guo T, Fan Y, Chen M, Wu X, Zhang L, He T, Wang H, Wan J, Wang X, Lu Z. Cardiovascular Implications of Fatal Outcomes of Patients With Coronavirus Disease 2019 (COVID-19). JAMA Cardiol. 2020 Mar 27;5(7):1-8. doi: https:/doi. org/10.1001/jamacardio.2020.1017. Epub ahead of print. PMID: 32219356; PMCID: PMC7101506. Bai T, Tu S, Wei Y, Xiao L, Jin Y, Zhang L, et al. Clinical and Laboratory Factors Predicting the Prognosis of Patients with COVID-19: An Analysis of 127 Patients in Wuhan, SSRN, 2020 DOI: https:// doi.org/10.2139/ssrn.3546118

6. Ruan Q, Yang K, Wang W, Jiang L, Song J. Clinical predictors of mortality due to COVID-19 based on an analysis of data of 150 patients from Wuhan, China. Intensive Care Med. 2020;46(5):846-848. DOI: https://doi.org/10.1007/s00134-020-05991-x. Erratum in: Intensive Care Med. 2020 ABr 6.

7. Cheng Y, Luo R, Wang K, Zhang M, Wang Z, Dong L, Li J, Yao Y, Ge S, Xu G. Kidney disease is associated with in-hospital death of patients with COVID-19. Kidney Int. 2020;97(5):829-838. DOI: https://doi.org/10.1016/j.kint.2020.03.005

8. Ammirati E, Wang DW. SARS-CoV-2 inflames the heart. The importance of awareness of myocardial injury in COVID-19 patients. Int J Cardiol. 2020;311:122-123. DOI: https://doi.org/10.1016/j.ijcard.2020.03.086

9. Zhou F, F, Yu T, Du R, Fan G, Liu Y, Liu Z, et al. Clinical course and risk factors for mortality of adult inpatients with COVID-19 in Wuhan, China: a retrospective cohort study. Lancet. 2020;395(10229):1054-1062. DOI: https://doi. org/10.1016/S0140-6736(20)30566-3. Erratum in: Lancet. 2020 Mar 28;395(10229):1038. Erratum in: Lancet. 2020 Mar 28;395(10229):1038.

10. Zhang G, Zhang J, Wang B, Zhu X, Wang Q, Qiu $S$, et al. Analysis of clinical characteristics and laboratory findings of 95 cases of 2019 novel coronavirus pneumonia in Wuhan, China: a retrospective analysis. Respir Res. 2020; 21(74). https://doi. org/10.1186/s12931-020-01338-8

11. Wang F, Hou H, Luo Y, Tang G, Wu S, Huang M, et al. The laboratory tests and host immunity of COVID-19 patients with different severity of illness. JCI Insight. 2020;5(10):e137799. doi: https://doi. org/10.1172/jci.insight.137799.

12. Liu K, Fang YY, Deng Y, Liu W, Wang MF, Ma JP, et al. Clinical characteristics of novel coronavirus cases in tertiary hospitals in Hubei Province. Chin Med J (Engl). 2020;133(9):1025-1031. DOI: https://doi. org/10.1097/CM9.0000000000000744

13. Mardani R, Vasmehjani A, Zali F, Gholami A, Nasab S, Kaghazian H, et al. Laboratory Parameters in Detection of COVID-19 Patients with Positive RT-PCR; a Diagnostic Accuracy Study. Mardani R, Ahmadi Vasmehjani A, Zali F, et al. Laboratory Parameters in Detection of COVID-19 Patients with Positive RT-PCR; a Diagnostic Accuracy Study. Arch Acad Emerg Med. 2020;8(1): e43

14. Herold T, Jurinovic V, Arnreich C, Lipworth B, Hellmuth J, Bergwelt-Baildon M, et al. Elevated levels of IL- 6 and CRP predict the need for mechanical ventilation in COVID-19. J Allergy Clin Immunol. 2020;146(1):128-136.e4. DOI: https://doi.org/10.1016/j.jaci.2020.05.008

15. Wei YY, Wang RR, Zhang DW, et al. Risk factors for severe COVID-19: Evidence from 167 hospitalized patients in Anhui, China. J Infect. 2020;81(1):e89-e92. DOI: https://doi.or$\mathrm{g} / 10.1016 / j . j i n f .2020 .04 .010$

16. Xu, Z, Shi L, Wang Y, Zhang J, Huang L, Zhang C, et al. Pathological findings of COVID-19 associated with acute respiratory distress syndrome. Lancet Respir. Med. 2020;8(4):420-422. DOI: https://doi. org/10.1016/S2213-2600(20)30076-X

17. Siordia JA Jr. Epidemiology and clinical features of COVID-19: A review of current literature. J Clin Virol. 2020;127:104357. DOI: https://doi.org/10.1016/j.jcv.2020.104357

18. W.J. Guan, Z.Y. Ni, Y. Hu, et al., Clinical characte- 
ristics of 2019 novel coronavirus infection in China, N Engl J Med 2020;382:1708-1720. DOI: https:// doi.org/10.1056/NEJMoa2002032

19. Li Q, Ding X, Xia G, Geng Z, ChenF, Wang L, Wang Z. A simple laboratory parameter facilitates early identification of COVID-19 patients. Medxiv.2020. Disponible en: https://www.medrxiv. org/content/10.1101/2020.03.17.20037572v1

20. Liu Y, Yang Y, Zhang C, Huang F, Wang F, Yuan J, et al. Clinical and biochemical indexes from 2019$\mathrm{nCoV}$ infected patients linked to viral loads and lung injury. Sci. China Life Sci. 2020; 63: 364-374. DOI: https://doi.org/10.1007/s11427-020-1643-8

21. Aboughdir M, Kirwin T, Abdul Khader A, Wang B. Prognostic Value of Cardiovascular Biomarkers in COVID-19: A Review. Viruses. 2020;12(5):527. DOI: https://doi.org/10.3390/v12050527

22. Vetter P, Vu DL, L'Huillier A, Schibler M, Kaiser L, Jacquerioz F. Clinical features of covid-19 The wide array of symptoms has implications for the testing strategy. BMJ. 2020; 369:m1470. DOI: https://doi. org/10.1136/bmj.m1470

23. Chan F, Yuan S, Kok K, To K, Chu H, Xing J, et al. A familial cluster of pneumonia associated with the 2019 novel coronavirus indicating person-to-person transmission: study of a family cluster. Lancet 2020; 395: 514-23. DOI: https://doi.org/10.1016/ S0140-6736(20)30154-9

24. Chen N, Zhou M, Dong X, Qu J, Gong F, Han Y, et al. Epidemiological and clinical characteristics of 99 cases of 2019 novel coronavirus pneumonia in Wuhan, China: a descriptive study. Lancet 2020; 395: 507-13. DOI: https://doi.org/10.1016/S01406736(20)30211-7

25. Inciardi R, Lupi L, Zaccone G, Italia L, Raffo M, Tomasoni D, et al. Cardiac Involvement in a Patient with Coronavirus Disease 2019 (COVID-19). JAMA Cardiol. 2020. DOI: https://doi.org/10.1001/jamacardio.2020.1096

26. Wang D, Hu B, HuC, Zhu F,Liu X, Zhang J, et al.
Clinical Characteristics of 138 Hospitalized Patients with 2019 Novel Coronavirus-Infected Pneumonia in Wuhan, China. JAMA. 2020;323(11):1061-1069. DOI: https://doi.org/10.1001/jama.2020.1585

27. Wan, S.; Xiang, Y.; Fang,W.; Zheng, Y.; Li, B.; Hu, Y.; Lang, C.; Huang, D.; Sun, Q.; Xiong, Y.; et al. Clinical Features and Treatment of COVID-19 Patients in Northeast Chongqing. J. Med. Virol. 2020;92(7):797-806. DOI: https://doi. org/10.1002/jmv.25783.

28. Jin YH, Cai L, Cheng ZS, Cheng H, Deng T, Fan YP, et al. A rapid advice guideline for the diagnosis and treatment of 2019 novel coronavirus (2019-nCoV) infected pneumonia (standard version). Mil Med Res. 2020;7(1):4. DOI: https://doi.org/10.1186/ s40779-020-0233-6

29. McRae M, Simmons G, Christodoulides N, Lu Z, Kang S, Fenyo D, et al. Clinical decision support tool and rapid point-ofcare platform for determining disease severity in patients with COVID-19. Lab Chip. 2020; 20:2075-85. DOI: https://doi. org/10.1039/d0lc00373e.

30. Tang N, Li D, Wang X, Sun Z. Abnormal coagulation parameters are associated with poor prognosis in patients with novel coronavirus pneumonia. J. Thromb. Haemost. 2020; 18: 844-847. DOI: https://doi.org/10.1111/jth.14768

31. Lippi G, Favaloro EJ. D-dimer is Associated with Severity of Coronavirus Disease 2019: A Pooled Analysis. Thromb Haemost. 2020;120(5):876-878. doi: https://doi.org/10.1055/s-0040-1709650

32. Zhang L, Yan X, Fan Q, Lui H, Liu X, Liu Z, et al. D-dimer levels on admission to predict in-hospital mortality in patients with Covid-19. J Thromb Haemost. 2020;18(6):1324-1329. DOI: https:// doi.org/10.1111/jth.14859

33. Bavishi C, Maddox TM, Messerli FH. Coronavirus Disease 2019 (COVID-19) Infection and Renin Angiotensin System Blockers. JAMA Cardiol. 2020. DOI: https://doi.org/10.1001/jamacardio. 2020.1282 
34. Petrilli CM, Jones SA, Yang J, Rajagopalan H, O’Donnell L, Chernyak Y, et al. Factors associated with hospital admission and critical illness among 5279 people with coronavirus disease 2019 in New York City: prospective cohort study. BMJ. 2020;369:m1966. DOI: https://doi.org/10.1136/ bmj.m1966

35. Holshue M, DeBolt C, Lindquist S, Lofy K, Wiesman J. Bruce H, et al. First Case of 2019 Novel Coronavirus in the United States. N Engl J Med. 2020; N Engl J Med 2020; 382:929-936. DOI: https:// doi.org/10.1056/NEJMoa2001191

36. Xu L, Liu J, Lu M, Yang D, Zheng X. Liver injury during highly pathogenic human coronavirus infections. Liver Int. 2020;40(5):998-1004. DOI: https://doi.org/10.1111/liv.14435

37. Arentz M, Yim E, Klaff L, Lokhandwala S, Riedo F, Chong F, et al. Characteristics and Outcomes of 21 Critically Ill Patients With COVID-19 in Washington State JAMA. 2020;; 323(16):1612-1614. DOI: https://doi.org/10.1001/jama.2020.4326

38. Wu J, Song S, Cao HC, Li LJ. Liver diseases in COVID-19: Etiology, treatment and prognosis. World J Gastroenterol. 2020;26(19):2286-2293. DOI: https://doi.org/10.3748/wjg.v26.i19.2286

39. Hui DSC, Zumla A. Severe Acute Respiratory Syndrome: Historical, Epidemiologic, and Clinical Features. Infect Dis Clin North Am. 2019;33(4):869-889. DOI: https://doi.org/10.1016/j.idc.2019.07.001

40. Lippi G, Plebani M. Laboratory abnormalities in patients with COVID-2019 infection. Clin Chem Lab Med. 2020;58(7):1131-1134. DOI: https://doi. org/10.1515/cclm-2020-0198

41. Xu XW, Wu XX, Jian XG, Xu KJ, Ying LJ, Ma CL, Clinical findings in a group of patients infected with the 2019 novel coronavirus (SARS-Cov-2) outside of Wuhan, China: retrospective case series. BMJ 2020.; 368 DOI: https://doi.org/10.1136/bmj.m606

42. Lippi G, Plebani M. Procalcitonin in patients with severe coronavirus disease 2019 (COVID-19): A meta-analysis. Clin Chim Acta. 2020;505:190-191. DOI: https://doi.org/10.1016/j.cca.2020.03.004

43. Fu, L., Wang, B., Yuan, T., Chen, X., Ao, Y., Fitzpatrick, T., et al. Clinical characteristics of coronavirus disease 2019 (COVID-19) in China: A systematic review and meta-analysis. The Journal of infection. 2020;80(6):656-665. DOI: https://doi. org/10.1016/j.jinf.2020.03.041

44. Harapan H, ItohN, Yufika A, Winardi W, Keam S, Te H. Coronavirus disease 2019 (COVID-19): A literature review. J Infect Public Health. 2020;13(5):667-673. DOI: https://doi.org/10.1016/j.jiph.2020.03.019.

45. L. Yan, H.T. Zhang, J. Goncalves, et al. A machine learning-based model for survival prediction in patients with severe COVID-19 infection, MedRxiv. 2020. DOI: https://doi.org/10.1101/2020.02.27.2 0028027. 